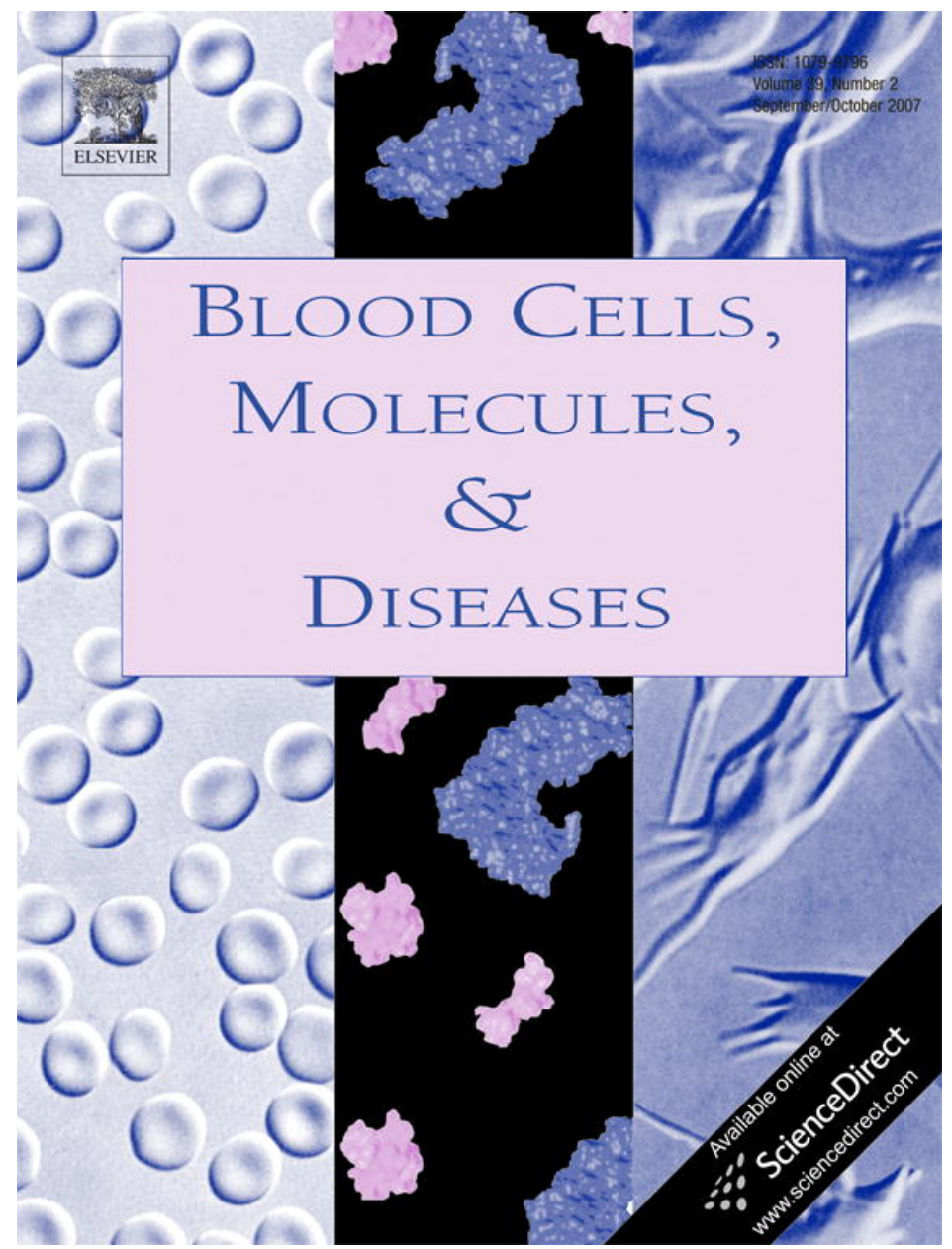

This article was published in an Elsevier journal. The attached copy

is furnished to the author for non-commercial research and education use, including for instruction at the author's institution, sharing with colleagues and providing to institution administration.

Other uses, including reproduction and distribution, or selling or licensing copies, or posting to personal, institutional or third party websites are prohibited.

In most cases authors are permitted to post their version of the article (e.g. in Word or Tex form) to their personal website or institutional repository. Authors requiring further information regarding Elsevier's archiving and manuscript policies are encouraged to visit: 
BloOd CELls, MOLECUlES, \& DISEASES

Blood Cells, Molecules, and Diseases 39 (2007) 156-159 www.elsevier.com/locate/ybcmd

\title{
Trisomy of chromosome 21 in leukemogenesis
}

\author{
Shai Izraeli*, Liat Rainis, Libi Hertzberg, Gil Smooha, Yehudit Birger \\ Research Section of Childhood Malignancies, Department of Pediatric Hemato-Oncology, and the Sheba Cancer Research Center, \\ Sheba Medical Hospital, Tel-Hashomer, Ramat-Gan 52621, Israel \\ Sackler Medical School, Tel-Aviv University, Israel \\ Submitted 16 April 2007 \\ Available online 29 May 2007 \\ (Communicated by M.A. Lichtman, M.D., 25 April 2007)
}

\begin{abstract}
Extra copies of chromosome 21 are often found in sporadic leukemias. Constitutional trisomy 21 of Down syndrome (DS) is associated with markedly increased risk for childhood leukemia. Thus the oncogenic role of trisomy 21 in the more common sporadic childhood leukemias may be revealed through the investigations of the relatively rare leukemias of DS. Recent studies of the megakaryoblastic leukemias of Down syndrome have uncovered a developmental leukemogenic mechanism characterized by a unique pre-natal collaboration between overexpressed genes from chromosome 21 and an acquired mutation in the transcription factor GATA1. The base of the markedly enhanced risk for acute lymphoblastic leukemia conferred by trisomy 21 is still unclear. Studies of the leukemias of DS are likely to contribute to the general understanding of the oncogenic mechanisms of chromosomal aneuploidies, the most common abnormalities in cancer.
\end{abstract}

(C) 2007 Elsevier Inc. All rights reserved.

Keywords: Down syndrome; Chromosome 21; Childhood leukemia; Megakaryoblastic leukemia; GATA1; Acute lymphoblastic leukemia

\section{Introduction}

The accumulation of multiple genetic abnormalities, or "hits", characterizes the evolution of the cancer cell. This genomic instability is commonly manifested by structural or numerical chromosomal aberrations [1]. Structural genomic aberrations leading to the activation of oncogenes or elimination of tumor suppression genes have been studied extensively. However, very little is known about the oncogenic role of numerical chromosomal aberrations, aneuploidy, which are the most common abnormalities in cancer.

\section{Aneuploidy of chromosome 21 and acute megakaryocytic leukemia}

Three (trisomy) or four (tetrasomy) copies of chromosome 21 is common in childhood acute lymphoblastic leukemia (ALL) [2-4]. Children with Down syndrome (DS) who carry

\footnotetext{
* Corresponding author. Research Section of Childhood Malignancies, Department of Pediatric Hemato-Oncology, and the Sheba Cancer Research Center, Sheba Medical Hospital, Tel-Hashomer, Ramat-Gan 52621, Israel. Fax: +97235303031.

E-mail address: sizraeli@sheba.health.gov.il (S. Izraeli).
}

trisomy 21 in all their cells have a 20 -fold increased risk for childhood ALL and 600-fold risk for acute megakaryocytic leukemia (AMKL) [5]. DS is not a classic genomic instability syndrome as the general risk for cancer is lower in these patients [5]. Rather, the high incidence of childhood leukemia in children with DS strongly suggests that additional copies of chromosome 21 are leukemogenic. Hence, studies of the pathogenesis of leukemia in patients with DS may clarify the role of acquired trisomy 21 in sporadic leukemias.

Approximately $10 \%$ of children with DS are born with a clonal megakaryocytosis syndrome commonly called "transient myeloproliferative disorder" (TMD) or "transient abnormal myelopoiesis" (TAM) or "transient leukemia". As suggested by the different names the disorder is usually transient and resolves spontaneously within up to several months. The biological mechanism of the spontaneous resolution is unclear. About $1-2 \%$ of DS patients will develop, however, a full-blown malignant acute megakaryoblastic leukemia (AMKL) during their first 4 years of life that will not regress without chemotherapy. In fact, the risk of AMKL is about 600 times higher in children with DS [5]. The factor(s) underlying the transformation from "benign" TMD into "malignant" AMKL are largely unknown. 
Thus the megakaryocytic malignancies of DS provide a "natural genetic model" of multistep lineage-specific leukemogenesis. Both the congenital disorder and the full-blown AMKL are characterized by differentiation arrest of the megakaryocytic lineage. The peculiar association between DS and childhood megakaryoblastic disorders has led to intensive search for gene or genes on chromosome 21 than may cause the differentiation arrest and initiate the leukemia. A surprising twist in this story came with the discovery that a gene on chromosome $X$, GATA1, was mutated in the megakaryoblasts from all the patients with DS and either TMD or AMKL [6,7]. The mutations were also found in fetal liver of aborted DS fetuses [8]. The mutations are acquired as they are not found in remission samples, and are specific to the megakaryoblastic disorders associated with trisomy 21 . Thus a clear model for multistep leukemogenesis in DS emerges: In a relatively high proportion of DS patients, acquired mutations in GATA1 are selected in utero and are probably responsible for the differentiation arrest and the initiation of clonal proliferation of immature megakaryoblasts. These mutations are necessary but insufficient for the development of the full-blown AMKL that affects some of these patients during early childhood (Fig. 1).

GATA1 encodes a zinc-finger transcription factor that regulates the normal development of the erythroid, megakaryocytic and basophilic/mast cell lineages. Mice lacking GATA1 expression in the megakaryocytic lineage have thrombocytopenia and extensive proliferation of immature megakaryoblasts. Inherited inactivating mutations in GATA1 in humans cause a familial dyserythropoietic anemia and thrombocytopenia [9]. Thus GATA1 normally suppresses the proliferation of megakaryocytic and erythroid precursors while promoting their differentiation. Two isoforms of GATA1 are usually detected: a full length GATA1 translated from the first ATG on exon 2, and a shorter form (GATA1s) that is initiated from an ATG on exon 3. The normal function of GATA1s is unknown. Presumably the balance between these two products serves a regulatory function in normal megakaryocytic development. All the acquired mutations in the megakaryoblastic disorders of DS result in elimination of the full-length GATA1 and the preservation of GATA1s.

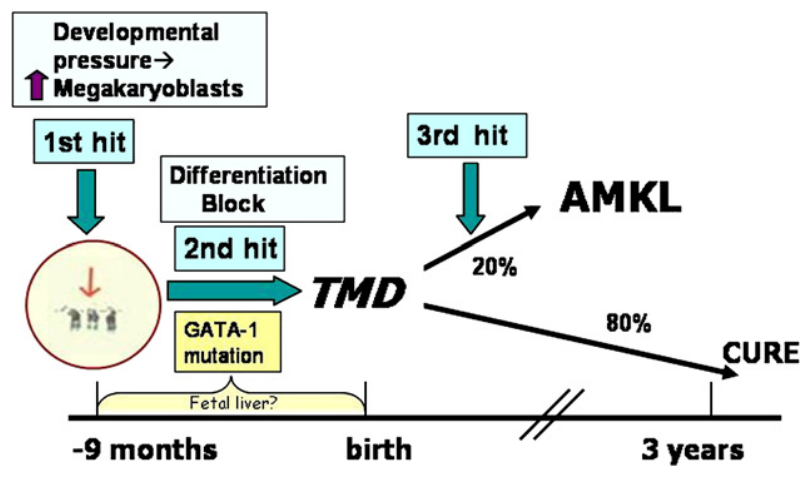

Fig. 1. A model for leukemia development in patients with Down syndrome (DS): trisomy 21 creates a pro-megakaryopoiesis developmental pressure during fetal hematopoiesis. Acquired mutation in GATA1 (GATA1s) causes differentiation arrest and enhanced proliferation of megakaryoblastic precursors leading to congenital leukemia (TMD): the evolution of this transient congenital leukemia to full-blown acute megakaryocytic leukemia (AMKL).
Because in DS the trisomy 21 is constitutional, i.e. it resides in every cell, it may promote leukemia in a non-autonomous manner. Possibly, the presence of trisomy 21 in non-hematopoietic "stromal" cells in the fetal liver may change the microenvironment and support the proliferation of the special fetal hematopoietic progenitors that are sensitive to GATA1s. This hypothesis may also explain why the transient megakaryoblastic proliferation resolves after birth. However, since very little in known about the regulation of fetal liver hematopoiesis, this hypothesis is currently difficult to study.

Alternatively, increased expression of certain chromosome 21 genes in the same cell that carries the GATA1s mutation may enhance its proliferation or survival ("a cell autonomous" hypothesis). We have recently demonstrated the potential involvement of ERG, an ets transcription factor on chromosome $21 \mathrm{q}$, in the DS megakaryocytic leukemias [10]. ERG is a protooncogene that is rarely involved in AMKL caused by the ERGTLS translocations. We have shown that it is expressed in CD34 cells, in normal megakaryocytes and platelets, in megakaryocytic leukemias (whether or not associated with DS), but not in normal or malignant erythroblasts. ERG is induced upon megakaryocytic differentiation of erythroleukemia cells. Forced expression of ERG in an erythroleukemia cell line causes a phenotypic shift from the erythroid into the megakaryocytic lineage. In recent unpublished experiments from our laboratory, we show that knockdown of ERG in a megakaryoblastic leukemia cell lines reverse their megakaryoblastic phenotype. Together these observations suggest that ERG is a positive regulator of normal and malignant megakaryopoiesis. We are currently testing the collaboration between $E R G$ and GATA1s mutation in mouse models.

A trisomy, i.e. the addition of a whole copy of a chromosome, is fundamentally different from an amplification of a small chromosomal segment containing one or more genes. We have recently showed that trisomies result in elevated expression of multiple genes residing on the trisomic chromosome. Generally the change of the level of expression corresponds to the genomic copy number. Indeed it is possible to deduce the presence of chromosomal trisomies from the gene expression pattern [11]. This pattern of gene expression raises the hypothesis that the oncogenic activity of trisomies may be conferred by a collaboration of several mildly overexpressed genes residing on the trisomic chromosomes.

Indeed there are several genes on the small chromosome 21 that encode proteins that may enhance megakaryopoiesis. These genes include RUNX1 [12,13], Ets2 and a newly discovered group of genes coding for regulatory micro-RNAs. For example both miR99 $a$ and miR125b are overexpressed in DS AMKL and may be associated with megakaryopoiesis [14] (and unpublished data from our laboratory). These observations led us to propose a developmental "rush hour" model for the occurrence of megakaryocytic leukemias in DS [10]. We suggest that mild overexpression of several genes on chromosome 21 create a positive pressure towards megakaryopoiesis, similarly to the traffic pressure towards downtown during rush hour. Supporting this suggested enhanced fetal megakaryopoiesis is the observation that normal DS infants have significantly higher platelets counts during the first 6 months of life [15]. Moreover, striking unpublished 
observations from Irene Roberts Laboratory (presented in the recent meeting of the American Society of Hematology, Dec. 2006) suggest a markedly enlarged megakaryocyte-erythroid progenitor department in human DS fetal livers. Together, these observations support our model that overexpressed genes from trisomy 21 promotes fetal megakaryopoiesis and "sets the stage" for a differentiation arresting mutations.

In our proposed model, the GATA1s mutation is similar to a "traffic accident" - it prevents megakaryopoiesis from reaching the target - platelet formation and further enhances the proliferation of a putative fetal megakaryocytic precursor, as shown by $\mathrm{Li}$ et al. [16]. The consequence is a pile-up of megakaryocytic precursors. In contrast to the car traffic-jam, only megakaryocytic progenitors with the GATA1s accumulate. This clonal accumulation results in the congenital leukemic phenotype.

\section{Implications of DS megakaryocytic leukemias, to the studies of childhood leukemias}

First, it is a prime example of collaborating pro-proliferation and differentiation arresting mutations in leukemia. Second, it may provide a general model for the role of aneuploidy in leukemia. Unique to whole chromosome aneuploidy is the parallel amplification of multiple genes which may act cooperatively in the same leukemogenic pathway [11]. Finally, deciphering the regulatory factors working towards the selection of GATA1s progenitors during fetal liver hematopoiesis in DS may be relevant to sporadic childhood leukemia since recent studies have clarified that most, if not all, childhood leukemia arise during fetal hematopoiesis [17].

\section{DS and acute lymphoblastic leukemia}

The megakaryocytic leukemia of Down syndrome is a unique disease [18]. However patients with Down syndrome are also at a markedly increased risk for childhood acute lymphoblastic leukemias (ALL). Because tri- or tetrasomy of chromosome 21 are the most commonly acquired chromosomal abnormalities in ALL, the study of DS ALL may have direct implications for sporadic childhood ALL. In most published multi-institutional ALL protocols, DS ALL comprise about $1-3 \%$ of total patients [19-21]. The age distribution and the immunophenotype are similar to "common ALL". "Common ALL" is a B-cell precursor leukemia that occurs most commonly in young preschool children.

ALL may be caused by a direct oncogenic effect of trisomy 21 , similarly to the role of additional chromosomes 21 in sporadic leukemias. Alternatively the effect of trisomy 21 may be developmental. Similarly to the suggested model for the megakaryocytic leukemias of DS, constitutional trisomy 21 may enhance the proliferation of a normal fetal lymphoid progenitor. This excess proliferation could evolve into leukemia if additional genetic events occur. Viral infections and the immunological response have long been suggested to have a role in the pathogenesis of childhood common ALL [22,23]. The markedly increased risk of ALL in DS could also be caused by the altered immunological environment and the increased infection rate that characterize DS.
Molecular epidemiology studies may clarify the leukemogenic role of constitutional trisomy 21. "Common" sporadic childhood ALL is usually associated with one of two genetic abnormalities: a structural chromosomal anomaly - fusing the AML1 (RUNX1) gene on chromosome 21 with the TEL (ETV6) gene on chromosome 12 , or a numerical abnormality hyperdiploidy [24]. These two genetic aberrations are mutually exclusive suggesting that each activates an oncogenic pathway leading to B-cell precursor leukemia. If trisomy 21 enhances the risk for childhood ALL indirectly we could expect a similar rate of secondary aberrations (hyperdiploidy or TEL/AML1 translocation) to sporadic common ALL. If, on the other hand, constitutional trisomy 21 has a direct leukemogenic effect similarly to the role of the acquired extra copies of chromosome 21 in hyperdiploid ALL, then we would expect a lower prevalence of TEL/AML1 or hyperdiploid genotypes in the ALL of Down syndrome.

There have been several studies summarizing the epidemiology of DS ALL during ALL protocols of the 1970s and 1980s as well as more recent reports by the COG and the NOPHO groups [19-21,25]. However, the number of DS ALL patients is still relatively small for any definite conclusions or subgroup analysis. For example, several studies demonstrated very low prevalence of TEL/AML1 translocations (consistent with the "direct role" hypothesis), while another has demonstrated the reverse [26]. Clearly an international molecular epidemiological study of DS ALL is warranted.

A recent genomic study on children with sporadic ALLs [27] as well as unpublished data from our laboratory on patients with DS reveal frequent monoallelic deletions of genes coding regulators of B cell development such as PAX5. Thus, similarly to the megakaryoblastic leukemias of DS, a collaboration between trisomy 21 and a differentiation arresting mutation in a lineage-specific transcription factor may also underline the development of lymphoblastic leukemias.

\section{Acknowledgments}

Funding for our research on chromosome 21 and leukemia is provided by the Israel Science Foundation, The Chief Scientist of the Israeli Health Ministry, The JNF UK, German Israel Foundation, Waxman Cancer Research Foundation (New York) and Mr Curtis Katz. I thank members from my group for their contribution as well as Drs Oskar Haas, Sabine Strehl, Peter Aplan, Hugh Brady, Owen Williams, Yoram Groner, Ditsa Levanon, Etsuro Ito, Berthold Gottgens, Eytan Domany, Gidi Rechavi for helpful discussions.

\section{References}

[1] A.V. Roschke, G. Tonon, K.S. Gehlhaus, N. McTyre, K.J. Bussey, S. Lababidi, D.A. Scudiero, J.N. Weinstein, I.R. Kirsch, Karyotypic complexity of the NCI-60 drug-screening panel, Cancer Res. 63 (2003) 8634-8647.

[2] E. Forestier, S. Heim, E. Blennow, G. Borgstrom, G. Holmgren, K. Heinonen, J. Johannsson, G. Kerndrup, M.K. Andersen, C. Lundin, A. Nordgren, R. Rosenquist, B. Swolin, B. Johansson, Cytogenetic abnormalities in childhood acute myeloid leukaemia: a Nordic series 
comprising all children enrolled in the NOPHO-93-AML trial between 1993 and 2001, Br. J. Haematol. 121 (2003) 566-577.

[3] C.J. Harrison, The detection and significance of chromosomal abnormalities in childhood acute lymphoblastic leukaemia, Blood Rev. 15 (2001) 49-59.

[4] M.E. Ross, X. Zhou, G. Song, S.A. Shurtleff, K. Girtman, W.K. Williams, H.C. Liu, R. Mahfouz, S.C. Raimondi, N. Lenny, A. Patel, J.R. Downing, Classification of pediatric acute lymphoblastic leukemia by gene expression profiling, Blood 102 (2003) 2951-2959.

[5] H. Hasle, Pattern of malignant disorders in individuals with Down's syndrome, Lancet Oncol. 2 (2001) 429-436.

[6] J. Wechsler, M. Greene, M.A. McDevitt, J. Anastasi, J.E. Karp, M.M. Le Beau, J.D. Crispino, Acquired mutations in GATA1 in the megakaryoblastic leukemia of Down syndrome, Nat. Genet. 32 (2002) 148-152.

[7] L. Rainis, D. Bercovich, S. Strehl, A. Teigler-Schlegel, B. Stark, J. Trka, N. Amariglio, A. Biondi, I. Muler, G. Rechavi, H. Kempski, O.A. Haas, S. Izraeli, Mutations in exon 2 of GATA1 are early events in megakaryocytic malignancies associated with trisomy 21, Blood 102 (2003) 981-986.

[8] J.W. Taub, G. Mundschau, Y. Ge, J.M. Poulik, F. Qureshi, T. Jensen, S.J. James, L.H. Matherly, J. Wechsler, J.D. Crispino, Prenatal origin of GATA1 mutations may be an initiating step in the development of megakaryocytic leukemia in Down syndrome, Blood 104 (2004) 1588-1589.

[9] J.D. Crispino, GATA1 in normal and malignant hematopoiesis, Semin. Cell Dev. Biol. 16 (2005) 137-147.

[10] L. Rainis, T. Toki, J.E. Pimanda, E. Rosenthal, K. Machol, S. Strehl, B. Gottgens, E. Ito, S. Izraeli, The proto-oncogene $E R G$ in megakaryoblastic leukemias, Cancer Res. 65 (2005) 7596-7602.

[11] L. Hertzberg, D.R. Betts, S.C. Raimondi, B.W. Schafer, D.A. Notterman, E. Domany, S. Izraeli, Prediction of chromosomal aneuploidy from gene expression data, Genes Chromosomes Cancer 46 (2007) 75-86.

[12] M. Ichikawa, T. Asai, T. Saito, G. Yamamoto, S. Seo, I. Yamazaki, T. Yamagata, K. Mitani, S. Chiba, S. Ogawa, M. Kurokawa, H. Hirai, AML-1 is required for megakaryocytic maturation and lymphocytic differentiation, but not for maintenance of hematopoietic stem cells in adult hematopoiesis, Nat. Med. 10 (2004) 299-304.

[13] K.E. Elagib, F.K. Racke, M. Mogass, R. Khetawat, L.L. Delehanty, A.N. Goldfarb, RUNX1 and GATA-1 coexpression and cooperation in megakaryocytic differentiation, Blood 101 (2003) 4333-4341.

[14] R. Garzon, F. Pichiorri, T. Palumbo, R. Iuliano, A. Cimmino, R. Aqeilan, S. Volinia, D. Bhatt, H. Alder, G. Marcucci, G.A. Calin, C.G. Liu, C.D. Bloomfield, M. Andreeff, C.M. Croce, MicroRNA fingerprints during human megakaryocytopoiesis, Proc. Natl. Acad. Sci. U. S. A. 103 (2006) 5078-5083
[15] S.M. Kivivuori, J. Rajantie, M.A. Siimes, Peripheral blood cell counts in infants with Down's syndrome, Clin. Genet. 49 (1996) 15-19.

[16] Z. Li, F.J. Godinho, J.H. Klusmann, M. Garriga-Canut, C. Yu, S.H. Orkin, Developmental stage-selective effect of somatically mutated leukemogenic transcription factor GATA1, Nat. Genet. 37 (2005) 613-619.

[17] M. Greaves, Pre-natal origins of childhood leukemia, Rev. Clin. Exp. Hematol. 7 (2003) 233-245.

[18] H. Hasle, C.M. Niemeyer, J.M. Chessells, I. Baumann, J.M. Bennett, G. Kerndrup, D.R. Head, A pediatric approach to the WHO classification of myelodysplastic and myeloproliferative diseases, Leukemia 17 (2003) 277-282.

[19] Y. Ravindranath, Down syndrome and leukemia: new insights into the epidemiology, pathogenesis, and treatment, Pediatr. Blood Cancer 44 (2005) 1-7.

[20] J.A. Ross, L.G. Spector, L.L. Robison, A.F. Olshan, Epidemiology of leukemia in children with Down syndrome, Pediatr. Blood Cancer 44 (2005) 8-12.

[21] B. Zeller, G. Gustafsson, E. Forestier, J. Abrahamsson, N. Clausen, J. Heldrup, L. Hovi, G. Jonmundsson, S.O. Lie, A. Glomstein, H. Hasle, Acute leukaemia in children with Down syndrome: a population-based Nordic study, Br. J. Haematol. 128 (2005) 797-804.

[22] U. Einav, Y. Tabach, G. Getz, A. Yitzhaky, U. Ozbek, N. Amariglio, S. Izraeli, G. Rechavi, E. Domany, Gene expression analysis reveals a strong signature of an interferon-induced pathway in childhood lymphoblastic leukemia as well as in breast and ovarian cancer, Oncogene 24 (2005) 6367-6375.

[23] M.F. Greaves, Aetiology of acute leukaemia, Lancet 349 (1997) 344-349.

[24] C.H. Pui, D. Campana, W.E. Evans, Childhood acute lymphoblastic leukaemia - current status and future perspectives, Lancet Oncol. 2 (2001) 597-607.

[25] M. Bassal, M.K. La, J.A. Whitlock, H.N. Sather, N.A. Heerema, P.S. Gaynon, L.C. Stork, Lymphoblast biology and outcome among children with Down syndrome and ALL treated on CCG-1952, Pediatr. Blood Cancer 44 (2005) 21-28.

[26] M. Steiner, A. Attarbaschi, M. Konig, H. Gadner, O.A. Haas, G. Mann, Equal frequency of TEL/AML1 ${ }^{+}$acute lymphoblastic leukemia in children with and without Down syndrome, Pediatr. Hematol. Oncol. 22 (2005) $11-16$.

[27] C.G. Mullighan, S. Goorha, I. Radtke, C.B. Miller, E. Coustan-Smith, J.D. Dalton, K. Girtman, S. Mathew, J. Ma, S.B. Pounds, X. Su, C.H. Pui, M.V. Relling, W.E. Evans, S.A. Shurtleff, J.R. Downing, Genome-wide analysis of genetic alterations in acute lymphoblastic leukaemia, Nature (2007). 